\title{
Departamento de Ginecologia e Obstetrícia da Faculdade de Medicina de Ribeirão Preto -USP Dados históricos sobre sua implantação e desenvolvimento
}

\author{
Geraldo Duarte; Roberto Salles Meirelles; Marcos Felipe Silva de Sá \& Rui Alberto Ferriani \\ Docentes. Departamento de Ginecologia e Obstetrícia. Faculdade de Medicina de Ribeirão Preto - USP
}

\section{CONSTITUIÇÃO DO CORPO DOCENTE E DOS SETORES ASSISTENCIAIS AO LON- GO DO TEMPO}

$\mathrm{E}$ $m$ fevereiro de 1956, o Prof. Zeferino Vaz convidou o Prof. Domingos Andreucci para dirigir a Cátedra da Clínica Obstétrica e Ginecológica da Faculdade de Medicina de Ribeirão Preto da Universidade de São Paulo (FMRP-USP). Desde a época de acadêmico, na Faculdade de Medicina de São Paulo, o Prof. Andreucci já pensava em ser ginecologista e obstetra, por isto, a organização e implantação do Departamento de Clínica Obstétrica e Ginecológica da FMRP-USP foi uma de suas grandes realizações. Seguindo seus impulsos e tendências naturais, o Prof. Andreucci deixou-se levar pelo fascínio da carreira universitária, tendo como objetivo formar médicos capazes, honestos e honrados, princípios que sempre adotou e que foram as bases das suas vitórias. Foi com tal espírito que nasceu o Departamento de Ginecologia e Obstetrícia dos dias atuais.

Inicialmente, as atividades do referido departamento foram desenvolvidas no Hospital das Clínicas da FMRP-USP e na Santa Casa de Ribeirão Preto. Naquela época, o Hospital das Clínicas funcionava na Maternidade Sinhá Junqueira, situada na Rua Bernardino de Campos, $\mathrm{n}^{\circ}$ 1000. Nesse endereço, atualmente, se encontra a Unidade de Emergência do Hospital das Clínicas. Como todo início de trabalho em uma estrutura médica universitária, a quota de sacrifícios foi enorme. No primeiro ano de funcionamento, as tarefas foram desenvolvidas com apenas dois Instrutores de Ensino, o Dr. José de Carvalho Guimarães e o Dr. Roberto Luiz Nogueira, trabalhando diuturnamente. Em março de 1957, foi contratado o Dr. Fedele Persio Emmanuele Renato Aristides Miraglia, como o terceiro Instrutor de Ensino do Departamento.

Após dois anos de atividades, o Prof. Andreucci deixou o departamento, sendo substituído pelo Prof. Bussâmara Neme, em maio de 1958. O Professor Neme chefiou o departamento apenas por seis meses (maio a novembro de 1958), tendo que regressar à Faculdade de Medicina de São Paulo, face aos compromissos universitários assumidos naquela faculdade, onde desenvolveu suas atividades até a aposentadoria. Hoje, o Prof. Neme é Professor Honorário naquela instituição.

Em novembro de 1958, também convidado pelo Prof. Zeferino Vaz, o Prof. Dr. Alberto Raul Martinez assumiu a Chefia do Departamento. No início, cuidou de assuntos que precisavam de soluções imediatas, tais como: reorganização de estrutura interna, escolha e designações dos Instrutores de Ensino, elaboração do programa didático, melhor aproveitamento dos 18 leitos de enfermaria, incentivar a parte científica em congressos, formação de pesquisadores, produção e publicações de trabalhos científicos. Assim, deu verdadeiro impulso ao "recém-nascido" departamento. Para tantas inovações e para o trabalho proposto, apenas três Instrutores de Ensino não eram suficientes, 
principalmente se levadas em conta as atividades assistenciais, desenvolvidas nas vinte e quatro horas diárias, sem considerar as atividades de ensino.

A saída do Dr. Fedele, em 1959, tornou mais evidente a necessidade de ampliação do quadro de Instrutores de Ensino para o Departamento de Ginecologia e Obstetrícia. Para solucionar esse impasse, o Prof. Alberto Raul Martinez procurou, entre os profissionais da época, jovens que estivessem seriamente interessados em trabalhar no regime de dedicação plena. Com tal política, foram distinguidos os Drs. Hemil Riscalla e Nelson Augusto, ambos formados na FMRP-USP e contratados em março de 1959. A primeira Reunião Clínica do Departamento foi realizada em 24/03/59 e dela participaram os Profs. Alberto Raul Martinez, José de Carvalho Guimarães, Nelson Augusto, Hemil Riscala e Victório Valeri.

Infelizmente, em 22 de abril de 1959, ocorreu a trágica morte do Dr. José de Carvalho Guimarães, uma grande perda em todos os sentidos, visto ser ele pessoa com aguçado espírito humanitário, profundo conhecedor da especialidade e auxiliar abnegado, servindo aos objetivos do departamento. Como já havia sido comprovado previamente, três instrutores não eram suficientes para cumprir todas as necessidades didáticas e assistenciais demandadas. Felizmente, no mesmo ano, os Drs. Manglio Fronzaglia, Norma Aparecida Mansoni Campos, Carlos Eduardo Martinelli e Ítalo Baruffi solicitaram estágio, amenizando a deficiência numérica de instrutores, principalmente para a assistência obstétrica. Atendendo aos apelos do Prof. Martinez para um crescimento sustentado do departamento, em setembro de 1959, a FMRP-USP contratou o Dr. Baruffi, importante aquisição acadêmica que se confirmou ao longo do tempo.

A partir de fevereiro de 1960, o Departamento de Clínica Obstétrica e Ginecológica passou a ser denominado Departamento de Obstetrícia e Ginecologia. Naquele mesmo ano, iniciou-se o Programa de Residência Médica com duração de dois anos na especialidade. Os primeiros residentes foram os Drs. Arnaldo Zanardi e Sérgio Bighetti, ambos formados pela FMRP-USP. Em março do mesmo ano, o Dr. Carlos Eduardo Martinelli foi contratado como Instrutor de Ensino, em regime de tempo integral. Mais tarde, fazendo jus à sua capacidade profissional e administrativa, foi eleito o Superintendente do Hospital das Clínicas da Faculdade de Medicina de Ribeirão Preto, cargo que exerceu por 12 anos. Em 1961, a
FMRP-USP contratou o Dr. Zanardi e o Dr. Bighetti, justamente os dois primeiros ex-residentes do departamento. O Dr. Bighetti dedicou-se à Oncologia Tocoginecológica e o Dr. Zanardi passou a auxiliar o Dr. Nogueira no Setor de Esterilidade Feminina.

A escassez de leitos e espaço para instalação e funcionamento dos Serviços Auxiliares foram, em parte, sanados com a inauguração da ala nova do Hospital das Clínicas. A ampliação beneficiou sobremaneira o setor, pois de 18 passou-se a contar com 45 leitos, número julgado suficiente para o ensino da especialidade às turmas de 70 alunos. Em 1961, o departamento contava com cinco residentes do $1^{\circ}$ ano: os Drs. Edward Marcolini, Maria Helena Cividanes, Jamil C. Muçouçah, Newton Lucas e Guido Iriarte Trigo. Em maio daquele ano, o Dr. Bighetti foi designado para estagiar no Serviço de Radioterapia do Hospital A.C. Camargo, com o objetivo de treinamento pessoal, visando à implantação futura do Setor de Radioterapia no Hospital das Clínicas da FMRP-USP.

Em 1962, o Prof. Martinez encontrava-se mais otimista, pois, sanadas as dificuldades relacionadas à assistência, teve mais tempo para dedicar-se com maior afinco, ao ensino médico e às atividades científicas. Nesse mesmo ano, o departamento passou a contar com mais um instrutor voluntário, o Dr. Rômulo Ribeiro da Costa. Quanto ao Programa de Residência Médica, o sucesso era marcado pela demanda. Além dos residentes do $2^{\circ}$ ano, o departamento contava com quatro residentes de $1^{\circ}$ ano, ou seja: os Drs. Jorge Antonio Salomão, Jesualdo Baldochi, José Renato Russo e Roberto Salles Meirelles. Na oportunidade, foram instalados os primeiros serviços auxiliares (Culdoscopia, Colposcopia, Colpocitologia, Laboratório de Endocrinologia, Diatermocoagulação, Esterilidade, Patologia da Mama e Roentgerapia). Cumpre destacar a preciosa colaboração do Dr. Vitório Valeri (Departamento de Morfologia) e do Dr. Hugo Brandão (Departamento de Patologia) ao trabalho do Prof. Ítalo Baruffi na criação dos Setores de Colposcopia e de Oncologia Tocoginecológica.

Em 1963, o departamento contava com sete instrutores efetivos, sete residentes e três instrutores voluntários. Em outubro daquele ano, a contratação do Dr. Roberto Salles Meirelles veio enriquecer sobremaneira o elenco de profissionais que faziam parte do departamento, auxiliando, de forma decisiva, a implantação futura do Programa de Pós-Graduação na FMRP-USP e no Departamento de Ginecologia e Obstetrícia. 
Nesse período, foi importante a colaboração científica do Dr. Carlos E. Negreiros de Paiva, Docente do Departamento de Fisiologia, no Setor de Fisiologia Obstétrica, dando início a uma profícua linha de pesquisa. Foi um período de atividades que tiveram extrema importância ao longo da história do departamento, na atuação dos Prof. Alberto Raul Martinez, Carlos Eduardo Martinelli, Roberto Salles Meirelles, Sérgio Pereira da Cunha, Francisco Mauad Filho, Luis Antonio Bailão, Geraldo Duarte e Antonio Alberto Nogueira. Estabeleceu-se, assim, o primeiro vínculo com a Escola de Fisiologia Obstétrica do Prof. Caldeyro Barcia, onde estagiara o Prof. Negreiros. Cumpre salientar que as atividades científicas, nas quais estiveram envolvidos não só os instrutores, mas, também, os residentes e médicos voluntários, tomaram impulso a partir dessa época. Quatro departamentos da área básica da FMRP-USP tiveram participação nos citados eventos: o de Morfologia, na pessoa do Prof. Victório Valeri, contribuindo no Setor de Oncologia, como colaborador do Prof. Baruffi; o de Fisiologia, na pessoa do Prof. Negreiros, e a equipe de Fisiologia Obstétrica, iniciando os estudos sobre contratilidade uterina; e os de Farmacologia e Bioquímica, nos quais os Profs. Martinez, Martinelli e Meirelles pesquisaram sobre o bradicininógeno no ciclo grávido-puerperal, contando com a colaboração dos Profs. Carlos R. Diniz e Ivan Fiore de Carvalho (Bioquímica) e o Prof. Maurício Rocha e Silva (Farmacologia). Na época, foram delineadas três linhas de pesquisa: $1^{\text {a }}$ - Farmacologia Tocoginecológica, com os Profs. Meirelles, Martinez, Diniz e Carvalho; $2^{\text {a }}$ - Fisiologia Obstétrica, com os Profs. Martinez, Negreiros, Martinelli, Nelson Augusto, Zanardi, Marcolini e Meirelles; $3^{\mathrm{a}}$ - Oncologia Tocoginecológica, com os Profs. Baruffi, Bighetti, Victório Valeri e Hugo Brandão.

Em 1964, após quatro anos de intensos esforços, foi montado parcialmente o Laboratório de Pesquisa do departamento com a participação direta do Prof. Meirelles e com a colaboração do Prof. Martinez. Sua otimização deve-se à FMRP-USP, ao Hospital das Clínicas e à Fundação de Amparo à Pesquisa do Estado de São Paulo (FAPESP), bem como à Maternidade Sinhá Junqueira. Esse foi um dos primeiros passos para satisfazer às exigências do tempo integral com dedicação exclusiva, por ser um local próprio para o desenvolvimento da pesquisa científica básica ou de aplicação clínica. Essa etapa culminou com a conclusão do Curso Avançado de Ciências Básicas, curso que objetivou a preparação de pessoal docente da
FMRP-USP para os cursos de Pós-Graduação, cujo conteúdo contemplava tópicos de Fisiologia, Farmacologia, Bioquímica, Biomecânica, Matemática e Bioestatística. O curso durou 3 anos e cada departamento da FMRP-USP designou um de seus docentes para treinamento. O representante do Departamento de Obstetrícia e Ginecologia foi o Prof. Meirelles.

Apesar do início das linhas de pesquisa assinaladas previamente, o ensino em Ginecologia e Obstetrícia foi essencialmente prático até a década de 60 , através de estágios nas enfermarias do Hospital das Clínicas e na Maternidade da Santa Casa de Ribeirão Preto. Em janeiro de 1964, o Dr. Jesualdo Baldochi foi contratado como Instrutor de Ensino, atividade que exerceu por dois anos. Nessa mesma época, também foi contratado o Dr. Edward Marcolini, como Instrutor Voluntário, que colaborou com o departamento até julho de 1966, sem remuneração.

Graças à maior compreensão dos órgãos competentes, o Departamento de Ginecologia e Obstetrícia pôde ampliar seu quadro, contratando mais três docentes, em 1965, os médicos Oswaldo Cruz Franco, Raimundo Sant'Ana e o Dr. Rômulo Ribeiro da Costa. Tais contratações possibilitaram sustentar a demanda crescente dos serviços especializados de assistência tocoginecológica, assim como publicar trabalhos científicos. Nessa oportunidade, foi criada a Residência de $3^{\circ}$ ano, iniciada a colaboração do departamento com a Escola de Enfermagem de Ribeirão Preto, sendo ministradas aulas para o Curso de Complementação Obstétrica e Ginecológica.

Na seqüência de importantes aquisições, em junho de 1966, o Departamento de Ginecologia e Obstetrícia ampliou seu quadro de docentes, contratando a Prof $^{a}$. Maria Matheus, a primeira docente do sexo feminino. O esforço e a dedicação da Prof ${ }^{a}$. Maria fizeram crescer o Setor de Esterilidade Feminina do Departamento, tanto do ponto de vista científico quanto assistencial. A coordenação do referido setor foi passada às suas mãos a partir de 1968. Na década de 70, profissionais andrologistas passaram a colaborar no setor. Dessa colaboração, destaca-se o Dr. Rui Yamasaki, participante do grupo até o momento. Dessas outras contribuições assistenciais da Prof ${ }^{a}$. Maria para o departamento, destacam-se a criação do Setor de Toxoplasmose e Gestação (posteriormente anexado ao Setor de Patologia Obstétrica e atualmente denominado Setor de Gestação de Alto Risco) e sua ajuda aos Setores de Patologia Obstétrica, Endocrinologia Tocoginecológica, Cirurgia Ginecológica e Planejamento Familiar. 
Na Reforma Universitária, ocorrida na Universidade de São Paulo, em 1969, foi definido o número mínimo necessário de docentes para se constituir um departamento, considerando-se, além do número total, o número mínimo de profissionais em cada categoria docente. Com tais alterações, o Departamento de Puericultura e Pediatria não preenchia os requisitos para continuar como Unidade Departamental, sendo necessário unir-se a outro departamento. $\mathrm{Na}$ época, a opção mais adequada foi a união com o Departamento de Obstetrícia e Ginecologia, criando-se o Departamento de Ginecologia, Obstetrícia e Pediatria, em 05/01/1970. A primeira reunião do conselho do novo departamento ocorreu em 11/03/1970, marco importante para demonstrar a organização departamental e o exercício da oportunidade de expressão democrática dentro da estrutura universitária.

$\mathrm{Na}$ prática, os resultados esperados com essa união de departamentos não se confirmaram. A possibilidade de execução de projetos conjuntos não se concretizaram, a despeito da aproximação científica entre os Setores de Neonatologia e Patologia Obstétrica. Apesar de haver certa independência, a falta de convergência entre os objetivos particulares de cada um dos antigos departamentos teve, como epílogo, a subdivisão departamental, que ocorreu em 07/12/1984. Após a subdivisão, o departamento passou a ser denominado Departamento de Ginecologia e Obstetrícia da Faculdade de Medicina de Ribeirão Preto da Universidade de São Paulo, sua designação atual.

A crescente necessidade de expansão do departamento levou o Prof. Martinez a solicitar nova ampliação do quadro de docentes à Reitoria, culminando com a liberação de três vagas em Regime de Dedicação Integral à Docência e à Pesquisa (RDIDP). A primeira vaga foi ocupada, em maio de 1970, pelo Prof. Sérgio Pereira da Cunha, docente que trouxe inúmeras contribuições para o departamento. Dentre elas, destaca-se a criação do Setor de Isoimunização Rh, embrião do atual Setor de Gestação de Alto Risco. A segunda vaga foi destinada à Prof ${ }^{a}$. Marta Edna H. Diógenes Yazlle, em junho de 1971, em resposta à imperativa necessidade do departamento de solidificar a interface junto à comunidade, tarefa que era parte do brilhante trabalho de Medicina Comunitária que a Prof ${ }^{a}$ Marta já desenvolvia no Centro Médico Social Comunitário de Vila Lobato. Posteriormente, a Prof ${ }^{a}$. Marta criou o Setor de Planejamento Familiar, vanguarda da moderna abordagem das ações anticonceptivas. Hoje, o setor é denominado Setor de Anti- concepção. Para a terceira vaga foi contratado o Dr. Silésio do Prado, em julho de 1971. Sua atuação foi mais dirigida para o Setor de Oncologia do Departamento, onde trabalhou até 1973.

A confiança da comunidade acadêmica da FMRP-USP na capacidade profissional do Prof. Martinez conduziu-o ao cargo de Diretor da Faculdade, de 1971 a 1975, e Superintendente do Hospital das Clínicas (04/05/1971-29/12/1971). A indicação para esses postos foi um reconhecimento ao seu potencial de trabalho e a sua disciplina, características marcantes da brilhante administração desenvolvida por ele. Com igual brilhantismo, dirigiu a Escola de Enfermagem de Ribeirão Preto, entre 1978 e 1982.

Em junho de 1972, o Prof. José Gonçalves Franco Júnior foi contratado como Docente do Departamento. Inicialmente, ele desenvolveu suas atividades de pesquisa junto ao Setor de Fisiologia e Farmacologia Tocoginecológica. Posteriormente, colaborou também com o Setor de Oncologia, até 1975, quando saiu do departamento.

Dentre os seis alunos matriculados no Programa de Pós-Graduação do departamento, no ano de 1972 (Frederico Pontes Gondim, Francisco Mauad Filho, Marcos Felipe Silva de Sá, Luiz Antonio Bailão José de Oliveira Couto Filho e Ademir Franzolim), os cinco primeiros foram contratados como docentes na FMRPUSP, deixando claro o papel fundamental do citado programa na formação de docentes para o próprio departamento desde a década de 70. Assim tem sido ao longo do tempo. No ano de 1973, o departamento contratou três docentes desse grupo. A primeira contratação foi a do Dr. Frederico Pontes Gondim, em setembro de 1973. Ele atuou no Departamento até 1977.

Em outubro de 1973, foi contratado o Prof. Marcos Felipe Silva de Sá, uma importante aquisição da academia e um dos atuais líderes do departamento. Seu dinamismo e conhecimento permitiram que continuasse o importante trabalho iniciado pelo Prof. Meirelles. Foi responsável pela expansão do Setor de Ginecologia Endócrina em todos os sentidos, na assistência, formação de pessoal, produção de ciência e administração. Foi, também, um dos responsáveis pela união entre os Setores de Ginecologia Endócrina e o de Infertilidade Conjugal, criando o sólido e produtivo Setor de Reprodução Humana, em 1988. Sua capacidade administrativa levou-o ao cargo de Superintendente do Hospital das Clínicas da FMRP-USP de 1994 até o momento, tarefa que vem desenvolvendo com brilhantismo. No mesmo mês de outubro, foi contra- 
tado o Prof. Francisco Mauad Filho, profissional dedicado aos ensinamentos obstétricos e idealizador do Setor de Cardiotocografia. Apesar de, inicialmente, atuar em vários setores do departamento, foi com a modernização e instituição dos vários recursos tecnológicos para avaliação da vitalidade fetal que o Prof. Mauad solidificou suas linhas de pesquisa, com profícua produção científica sobre o tema.

O Prof. Luiz Antonio Bailão foi contratado como docente em março de 1974, representando uma importante aquisição para o departamento. Dentre suas várias atividades, destacam-se a criação dos Setores de Ultra-Som e de Medicina Fetal, bem como sua diferenciada colaboração na criação do Setor de Gestação de Alto Risco, setores com os quais colaborou até 1985. Hoje, o Prof. Bailão dirige um centro privado de assistência e ensino de ultra-sonografia. Em junho de 1974, o Departamento de Ginecologia e Obstetrícia foi agraciado com nova vaga docente, para a qual foi contratado o Prof. José de Oliveira Couto Filho, que desenvolveu suas atividades no Setor de Oncologia até 1977.

Até 1975, o atendimento de gestantes de risco no departamento era prestado por setores isolados, de acordo com a complicação obstétrica ou intercorrente. Com a liderança do Prof. Sérgio Pereira da $\mathrm{Cu}-$ nha, Maria Matheus de Sala, Marta Edna e Marcos Felipe $o$ atendimento foi hierarquizado, criando-se o Setor de Patologia Obstétrica, hoje denominado Gestação de Alto Risco. Posteriormente, ao longo do tempo, tal setor contou com a colaboração dos Profs. Francisco Mauad, Antonio Alberto Nogueira, Geraldo Duarte, Aderson Berezowski e Reinaldo Rodrigues. Com o aumento de atividades específicas dentro dos diferentes setores, o Prof. Felipe e a Prof ${ }^{a}$. Maria Matheus passaram a se dedicar mais à Reprodução Humana, e a Prof ${ }^{a}$. Marta, aos Setores de Medicina Comunitária e ao de Anticoncepção

Em agosto de 1975, o Prof. Reinaldo Rodrigues foi contratado como docente. Suas atividades iniciais foram no Setor de Endocrinologia Ginecológica. Posteriormente, colaborou na criação do Setor de Cirurgia Ginecológica e, em 1996, passou a colaborar também com o Setor de Gestação de Alto Risco. No mês de outubro de 1975, a Prof ${ }^{\mathrm{a}}$ Glaci Ribeiro da Silva transferiu-se do Departamento de Farmacologia para o Departamento de Obstetrícia e Ginecologia, com o objetivo de colaborar nas atividades de pesquisa do Setor de Oncologia. Desenvolveu suas atividades até 1982, quando solicitou afastamento sem vencimentos para trabalhar na Universidade de Campinas, situação que perdurou até maio de 1986, quando ela solicitou a transferência definitiva para aquela universidade.

A necessidade de expansão do Setor de Oncologia Tocoginecológica e Mamária levou o Prof. Baruffi a solicitar mais uma vaga docente, que foi ocupada pelo Prof. Paulo Meyer de Paula Philbert, em dezembro de 1975. Nesse setor o Prof. Philbert vem desempenhando suas atividades até o momento, além de colaborar com o Setor de Cirurgia Ginecológica Geral e Uroginecologia. Nos últimos anos, o Prof. Philbert tem prestado ajuda inestimável na recuperação e no estímulo do aleitamento materno, ministrando cursos e auxiliando no treinamento de profissionais da saúde com interesse nesse campo.

O ingresso do Prof. Odilon Iannetta, como docente do departamento, foi em agosto de 1977, na vaga decorrente da saída do Dr. Frederico Pontes Gondim. Dentre as várias contribuições do Prof. Odilon, destaca-se a criação do Setor de Climatério, um dos mais renomados serviços do país, em atendimento à mulher no período do climatério, que ele coordena até hoje.

O Dr. Antonio Carlos Farah foi contratado, em junho de 1977, e atuou no Setor de Oncologia do Departamento de Ginecologia e Obstetrícia até agosto de 1978. Com sua saída, a vaga foi ocupada pelo Prof. Dr. Maurício Mesquita Sabino de Freitas, contratado em agosto de 1978. O Prof. Maurício desenvolveu suas atividades no setor até 1993 , época em que saiu da Oncologia e criou o Setor de Uroginecologia. Atualmente, o Prof. Maurício coordena os Setores de Uroginecologia e o de Cirurgia Ginecológica.

A partir de 1972, as atividades obstétricas, desenvolvidas sob a responsabilidade do departamento, na Santa Casa de Ribeirão Preto, foram totalmente transferidas para as instalações do Hospital das Clínicas da Faculdade de Medicina, à Rua Bernardino de Campos, $n^{\circ} .1000$. Em março de 1978, com a inauguração do novo Hospital das Clínicas, situado no Campus da Faculdade de Medicina, o departamento transferiu-se para as novas e amplas instalações. Antes da referida mudança, a área sob a jurisdição do departamento era de $1.500 \mathrm{~m}^{2}$, passando para $4.665,39 \mathrm{~m}^{2}$ nas novas instalações.

Considera-se a mudança do Hospital das Clínicas para o Campus Universitário da Faculdade de Medicina, em 1978, como marco importante na existência do departamento. Ela possibilitou a criação de novas instalações burocráticas, proporcionando maior espaço físico e facilidades para os docentes, criação de maior 
número de ambulatórios especializados, ampliação do número de leitos hospitalares e local privilegiado para a instalação do laboratório de pesquisa. As instalações físicas do novo Laboratório de Pesquisa e a contratação de técnicos especializados pela FMRP-USP e pelo Hospital das Clínicas, com o apoio da FAPESP, CNPq, FINEP e CAPES, propiciaram condições extremamente favoráveis para a execução dos projetos da área. Os projetos consolidaram a liderança nacional do Departamento de Ginecologia e Obstetrícia como um dos mais produtivos dentro da especialidade.

Em 1983, o Prof. Geraldo Duarte foi contratado como docente. Após cumprir os vários estágios exigidos para todo novo docente no departamento, o Prof. Geraldo uniu-se ao grupo de Patologia Obstétrica, formado, na época, pelos Profs. Sérgio Pereira da Cunha, Francisco Mauad Filho, Maria Matheus de Sala, Luis Antonio Bailão e Marta Edna Holanda Diógenes Yazzle. Desde essa época, vem dedicandose ao estudo das gestações de risco e das infecções tocoginecológicas, notadamente aquelas que acometem gestantes e apresentam a transmissão vertical em seu espectro fisiopatológico.

Em 1983, aposentou-se o Prof. Martinelli, pertencente ao grupo dos pioneiros da implantação do departamento. Na vaga, foi contratado o Prof. Rui Alberto Ferriani, aquisição que enriqueceu objetivamente o departamento. Também foi exigido do Prof. Rui o cumprimento de estágios nos vários setores do departamento. Após tal período, o Prof. Rui dedicou-se com maior afinco ao estudo de temas relacionados à Endocrinologia Ginecológica e Esterilidade Feminina, unindo-se ao grupo de profissionais já existente, composto pelos Profs. Marcos Felipe Silva de Sá, Roberto Salles Meirelles, Reinaldo Rodrigues e Maria Matheus de Sala.

No ano de 1984, o departamento foi agraciado com a criação de dois claros para vagas de docente. Em uma das vagas, o departamento solidificou importante aquisição, pois foi possível contratar o Prof. Antonio Alberto Nogueira, em julho de 1985. Inicialmente, o Prof. Nogueira se ligou aos profissionais do Setor de Patologia Obstétrica, ao qual está ligado até hoje, a despeito de seu diferenciado conhecimento em videoendoscopia ginecológica, atualmente. Na segunda vaga, em julho de 1985, foi contratado o Prof. Hélio Humberto Angotti Carrara, para desenvolver suas atividades junto ao Setor de Oncologia e Mastologia, além das atividades habituais, vem se dedicando ao estudo da reconstrução mamária, especialmente daquelas decorrentes de cirurgias oncológicas.
Do ponto de vista assistencial, a criação do Setor de Cirurgia Ginecológica, disciplinando o fluxo de pacientes e otimizando a taxa de internações e cirurgias realizadas, foi de grande importância tanto para o ensino quanto para a assistência. Para a organização do mencionado setor em 1985, o Prof. Geraldo contou com a ajuda inestimável dos Profs. Marcos Felipe Silva de Sá, Rui Alberto Ferriani, Reinaldo Rodrigues, Antonio Alberto Nogueira e Hélio Humberto Angotti Carrara.

Em 1985, aposentou-se o Prof. Alberto Raul Martinez, após 25 anos de dedicação ao Departamento de Ginecologia e Obstetrícia. Embora tenha sido o terceiro chefe a partir da criação do departamento, deve-se destacar que foi a partir de sua direção que o departamento adquiriu a base de infra-estrutura e os contornos acadêmicos, necessários para se transformar em referência nacional.

Em abril de 1986, foi contratado o Prof. Marcos Dias de Moura. A despeito de colaborar em vários setores do departamento, atendendo aos apelos de sua formação no Curso de Pós-Graduação, juntou-se aos Docentes do Setor de Reprodução Humana, onde vem prestando importante contribuição.

O Prof. Jurandyr Moreira de Andrade foi contratado em janeiro de 1988, em claro concedido pelo Reitor. Sua valiosa contribuição se faz presente em vários setores do departamento, mas foi no Setor de Oncologia Tocoginecológica e Mamária que teve oportunidade de solidificar suas linhas de pesquisa, iniciadas durante o Curso de Pós-Graduação.

Em 1991, o Prof. Lewis Joel Greene foi transferido do Departamento de Farmacologia para o Departamento de Ginecologia e Obstetrícia. Com ele, vieram a notável bagagem de conhecimento científico e a experiência editorial, acumuladas nos últimos anos, dando subsídios para a organização de uma das mais concorridos disciplinas do Programa de Pós-Graduação na Área de Tocoginecologia. Infelizmente, com a Reestruturação Departamental ocorrida na USP, em 2000, o Prof. Greene transferiu-se para o Departamento de Biologia Celular e Molecular e Bioagentes Patogênicos.

O avanço da ecografia e das possibilidades diagnósticas de alterações fetais indicaram a necessidade de que o Setor de Ultra-Sonografia fosse totalmente redimensionado para atender à nova demanda, fornecendo subsídios para suporte e embasamento de técnicas invasivas e não invasivas. Com tais argumentos, foi concedido mais um claro para docente, 
possibilitando a contratação do Prof. Aderson Tadeu Berezowski, em outubro de 1993. Dentre várias atividades, o Prof. Aderson, vem modernizando o setor, além de auxiliar no Setor de Gestação de Alto Risco.

Com a saída do Prof. Bailão, a vaga foi ocupada pela Prof ${ }^{a}$. Rosana Maria dos Reis, em junho de 1995. A colaboração da Prof ${ }^{a}$. Rosana é expressa em vários setores do departamento, mas é no Setor de Reprodução Humana que ela tem desenvolvido suas linhas de pesquisa, junto com os outros componentes do grupo.

Sem dúvidas, a década de 90 trouxe importantes mudanças no Departamento de Ginecologia e Obstetrícia, tanto na parte científica quanto na parte assistencial. A rapidez do progresso técnico e científico impôs um ritmo de mudanças e adequações muitas vezes difícil de ser assimilado pelo departamento. Houve a necessidade de criação de, pelo menos, 6 novos setores de assistência terciária (Setores de Medicina Fetal, Moléstias Infectocontagiosas em Ginecologia e Obstetrícia, Uroginecologia, Videoendoscopia Ginecológica, Laserterapia e Ginecologia Infanto-Puberal). O primeiro setor criado na década de 90 foi o de Medicina Fetal, sob a liderança do Prof. Bailão, em 1990. O Prof. Bailão foi auxiliado pelo Grupo de Gestação de Alto Risco e pelos Prof. João Pina Neto, Lúcia Martelli e Ester Ramos, docentes do Departamento de Genética.

Apesar da clara necessidade de disciplinar e hierarquizar o atendimento de mulheres portadoras de doenças infectocontagiosas, de interesse do tocoginecologista desde a década de 80, só em março de 1993, foi possível a criação do Setor de Moléstias Infectocontagiosas em Ginecologia e Obstetrícia, sob liderança do Prof.Geraldo Duarte e o apoio de forma decisiva, do Prof. Marcos Felipe Silva de Sá e do Prof. Sérgio Pereira da Cunha. Situação similar ocorreu com a formação do Setor de Videoendoscopia Ginecológica. O Prof. Antonio Alberto Nogueira, o idealizador do setor, já vinha trabalhando com endoscopia desde o início da década, mas, só em 1997, passou a desenvolver as atividades relativas à endoscopia, dentro de um cronograma que exigia espaço físico, salas cirúrgicas, leitos hospitalares e pessoal treinado. Também não foi diferente com a criação do Setor de Uroginecologia pelo Prof. Maurício Sabino. A incontinência urinária de esforço, que sempre foi abordada dentro do contexto de cirurgia ginecológica geral, e a necessidade de disciplinar o atendimento diagnóstico, clínico e instrumentalizado de mulheres portadoras dessas afecções, assim como a necessidade de assimilar novas modalidades terapêuticas, fizeram com que tal setor fosse criado.
O Setor de Atendimento à Adolescente foi idealizado pela Prof ${ }^{a}$. Marta Edna e desenvolvido no Centro Social e Comunitário de Vila Lobato, devido à premência de espaço físico no Hospital das Clínicas do Campus. O setor também foi criado em resposta à demanda por esse tipo de atendimento diferenciado. A demanda por atendimento especializado também foi o motivo que levou o Prof. Geraldo a criar o Setor de Laserterapia do Tracto Genital Inferior. Na sequiência, a Prof ${ }^{a}$. Rosana Reis criou o Setor de Ginecologia Infanto-Puberal, para o qual teve o apoio de todos os profissionais do Setor de Reprodução Humana. No Setor de Oncologia, em seus protocolos assistenciais, ganharam posição de destaque a adoção de cirurgias com menor potencial de mutilação e a quimioterapia mais efetiva.

Em 26/03/1993, nasceu a primeira criança brasileira, gerada a partir de um programa de fertilização in vitro (Técnica de ICSI), totalmente desenvolvido em Serviço Público de Saúde, sob os cuidados da equipe composta pelos Profs. Marcos Dias de Moura, Marcos Felipe Silva de Sá, Maria Matheus e Rui Alberto Ferriani. O evento assegurou ao Departamento de Ginecologia e Obstetrícia a liderança na Área de Reprodução Assistida entre as Instituições Universitárias Públicas do Brasil, visto que é um tipo de assistência habitualmente oferecido por instituições privadas. O setor foi novamente pioneiro, ao ser o primeiro, no Serviço Público, a instalar a técnica de injeção intracitoplasmática de gametas (ICSI), com o nascimento da primeira criança, em 1996. Hoje, mais de 400 crianças já nasceram em decorrência das técnicas de reprodução assistida, realizadas no Departamento de Ginecologia e Obstetrícia.

Também na década de 90, reformou-se o Centro Obstétrico do Campus, juntamente com a área do Setor de Reprodução Humana. Com a reforma, foram incorporados $114 \mathrm{~m}^{2}$ à área do Laboratório de Ginecologia e Obstetrícia, melhorando o desempenho do Setor de Reprodução Humana e do Setor de Obstetrícia, em decorrência da ampliação objetiva da área de procedimentos para reprodução assistida e da área de diagnóstico ultra-sonográfico. Além do controle ecográfico, do desenvolvimento folicular e da aspiração oocitária, nessa área de expansão, também são realizados os exames ecográficos de morfologia fetal e os procedimentos fetais invasivos. No final da década, toda a área de enfermarias do $8^{\circ}$ andar foi reformada, assim como a área administrativa do departamento, com inauguração do anfiteatro e modernização das outras salas de aula. 
No período de reforma do Centro Obstétrico, as atividades obstétricas, de baixo e médio risco foram transferidas para o $3^{\circ}$ andar da Unidade de Emergência, onde ficaram de 1992 até 1998. Com o retorno de parte das atividades obstétricas ao Hospital das Clínicas-Campus, houve maior integração do Setor de Neonatologia com o Setor de Gestação de Alto Risco. Entretanto, com o crescimento contínuo na assistência de gestantes de alto risco, demandando atendimento diferenciado, em qualidade e quantidade de atendimentos, tornou-se imperioso buscar alternativas para o ensino da obstetrícia de baixa complexidade. A exemplo do que já ocorrera no passado, o departamento fez nova parceria com a Fundação Sinhá Junqueira, que havia construído uma maternidade no Complexo Aeroporto, área que alberga um dos segmentos populacionais mais carentes de Ribeirão Preto. Essa maternidade, denominada Mater, foi totalmente projetada, de acordo com as necessidades, para prestar assistência obstétrica de baixo risco. Em 1998, celebrou-se novo contrato entre a FMRP-USP, Departamento de Ginecologia e Obstetrícia da FMRPUSP, Hospital das Clínicas e Fundação Sinhá Junqueira, para que os partos considerados de baixo risco fossem realizados naquela maternidade. Assim, o número de partos realizados por nossos alunos e residentes foi aumentado em função do atendimento feito em dois hospitais, prestando-se assistência dentro dos padrões de hierarquização do SUS.

O programa da Fundação de Apoio ao Ensino, Pesquisa e Assistência (FAEPA) do Hospital das Clínicas para aproveitar novos valores foi uma das mais valiosas iniciativas no sentido de manter o quadro docente em constante atualização. A rigidez burocrática para renovação do quadro, esta dependente da Universidade de São Paulo, não permite agilidade nesse sentido, pois a contratação de novos docentes só ocorre, quando existe perda objetiva do docente. Esse ciclo de aproveitamento máximo da experiência acumulada do profissional tem seus aspectos positivos, mas, no tocante à agilidade para absorção de novos e diferenciados valores, ela é negativa. Pelo programa da FAEPA, foram contratados o Prof. Francisco Candido dos Reis, ligado aos Setores de Oncologia Tocoginecológica e de Videoendoscopia Ginecológica, e a Prof ${ }^{\mathrm{a}}$. Maria Célia Mendes, ligada ao Setor de Medicina de Família e Ecografia. Sem dúvidas, foram duas aquisições de extrema importância para a instituição, com potencial inestimável de contribuição para o departamento.

Especial distinção deve ser atribuída aos Médi- cos Assistentes do Hospital das Clínicas e da FMRPUSP no auxílio para o cumprimento das tarefas docentes. Seu trabalho, freqüentemente não reconhecido como de especial valor, na realidade, complementa de forma admirável as limitações impostas pela Universidade de São Paulo, extremamente conservadora quanto à expansão do quadro funcional de docentes.

\section{CHEFIAS DO DEPARTAMENTO}

As Chefias do Departamento tiveram a seguinte seqüência: Prof. Domingos Andreucci: no período de 1956-1958; Prof. Dr. Bussamara Neme: de maio a novembro de 1958; Prof. Alberto Raul Martinez: de 1958-1969, de 1975-1979 e de 1982-1985; Prof. Ítalo Baruffi: de 1970-1975 e de 1979-1982. O Prof. Roberto Salles Meirelles dirigiu o Departamento de janeiro de 1985 a janeiro de 1989. Em 1989, foi reconduzido à Chefia do Departamento, permanecendo até janeiro de 1991. Em seguida, foi eleito o Prof. Marcos Felipe Silva de Sá para o biênio de 1991 a 1993. O Prof. Sérgio Pereira da Cunha foi eleito para o biênio 1993 1994, sendo reconduzido para mais dois anos na chefia. Em 1996, o Prof. Rui Alberto Ferriani iniciou seu primeiro mandato, sendo também reconduzido para mais dois anos. Em novembro de 2000, foi eleito o Prof. Geraldo Duarte para a chefia do Departamento no biênio 2001-2002.

\section{REGIMENTO INTERNO E AS COORDE- NADORIAS DO DEPARTAMENTO}

O Regimento Interno do Departamento de Ginecologia e Obstetrícia foi aprovado em 08/09/1986, na chefia do Prof. Dr. Roberto Salles Meirelles. O esquema de Coordenadorias para o Ensino, Pesquisa, Assistência e Administração agilizou sobremaneira o processo administrativo, visto que sua filosofia tem a descentralização de decisões como base. Os primeiros coordenadores foram: Ensino: Prof. Rui Ferriani; Pesquisa: Prof. Marcos Felipe; Assistência: Prof. Sérgio Cunha, Administração: Prof ${ }^{a}$ Marta Edna. O esquema de administração departamental ainda está em vigor na atualidade.

\section{PESSOAL ADMINISTRATIVO E DO LA- BORATÓRIO DO DEPARTAMENTO}

Foram secretárias do departamento: a $\mathrm{Srt}^{\mathrm{a}}$. Jenny S. Villandangos, de 1958 a 1983, a Srt ${ }^{a}$ Maria 
Lúcia do Carmo, de 1983 a 1991, e a Srt ${ }^{\mathrm{a}}$. Iara Maria Corrêa, de 1991 a 1997. Desta data até o momento, o secretário do Departamento é o Sr. Reinaldo Vicente Tavares.

Colaboraram, ou ainda fazem parte, na Secretaria e no Laboratório do departamento, as seguintes pessoas: Ana Lúcia de Azevedo, Célia Maria Almeida Coelho, Claire Tais Montiani Scandar, Cláudia Helena Approbato, Devanir Candido de Oliveira, Elettra Greene, Eliana Cristina D'Lacqua, Elza Sebastiana F. Leonardo, Ilza Alves de Rezende Mazzucatto, José Carlos Porto, Lídia Alexandre do Carmo, Lúcia Helena Alves dos Santos, Márcia Aparecida Castro Toledo, Maria Albina Verceze Bortolieiro, Maria Angela Martins Ruzzene, Maria Aparecida Carneiro Vasconcelos, Maria Aparecida Piccinato, Maria Auxiliadora Pádua Rosa, Maria Cristina Piccinato M. de Araújo, Marilda Hatsumi Yamada Dantas, Marlene Lúcio, Nair Bizão, Neuza Targa, Odete Pizarro, Renata Maria Rebouças Carnevali, Rosane Aparecida Cunha Casula, Sandra Aparecida Cavichiollo Vianna, Vera Lúcia de Faria e Veranice Mello Machado.

\section{OBJETIVOS E RESULTADOS RELACIO- NADOS AO ENSINO (GRADUAÇÃO E PÓS- GRADUAÇÃO), ASSISTÊNCIA E PESQUISA}

\subsection{Ensino de Graduação}

Entre 1956 e 1960, o Departamento de Ginecologia e Obstetrícia preocupou-se, inicialmente, em ministrar cursos teóricos e práticos, que viessem a contribuir para a formação do médico generalista, de futuros obstetras e ginecologistas ou do médico que fosse aprofundar seus conhecimentos em outra especialidade. A primeira aula foi ministrada aos alunos do $5^{\circ}$ ano em 14/03/56, sobre os temas: Ovulação, Captação Ovular, Fecundação, Transplante Ovular e Nidação. A aula foi ministrada pelo Prof. Domingos Andreucci, nas dependências do antigo Centro Médico, na Rua Tibiriçá. Com o tempo, e o progresso, as técnicas diagnósticas e terapêuticas tornaram-se mais complexas, exigindo um curso mais adequado ao mercado de trabalho. As mudanças relatadas anteriormente, necessárias para tal adaptação, justificam a expansão do departamento, ocorrida desde sua criação até os dias atuais.

A mudança curricular, ocorrida em 1993, foi uma resposta direta às citadas necessida- des, ampliando-se o internato também para os alunos do $5^{\circ}$ ano, o que exigiu mudanças expressivas na distribuição das atividades assistenciais de nosso departamento, para dar sustentação ao ensino. A otimização do Centro Médico Social e Comunitário de Vila Lobato e do Centro de Saúde-Escola, foi uma das mudanças necessárias, obrigando a ampliação do número de médicos assistentes tanto do Hospital das Clínicas como da Faculdade de Medicina. Com a hierarquização do Sistema Único de Saúde, as instalações da Unidade de Emergência passaram a ser prioritárias para atividades emergenciais mais complexas. Hoje, o atendimento médico do departamento obedece a uma estrutura hierarquizada com os níveis primário, secundário e terciário em sintonia, o que permite maior eficiência, com reflexos no ensino, pesquisa e na própria assistência. Atualmente, o departamento tem cinco disciplinas de graduação, distribuídas do $3^{\circ}$ ao $6^{\circ}$ ano, sendo de internato os dois últimos anos.

O número de alunos da graduação, que passaram pelo departamento, pode ser observado na Tabela I, onde está relacionado, também, o número daqueles que permaneceram no serviço para o Programa de Residência Médica na especialidade.

\subsection{Ensino de Pós-Graduação Lato Sensu}

A formação de Especialistas em Ginecologia e Obstetrícia teve início a partir da década de 60, com a implantação da Residência Médica. Daquela época até os dias atuais, houve, não só aumento substancial no número de especialistas, como, também, o desenvolvimento de programas e a diversificação das áreas de conhecimento, caracterizando o departamento como um importante pólo de formação de especialistas.

\begin{tabular}{|c|c|c|c|}
\hline Ano & $\begin{array}{c}N^{\circ} d e \\
\text { Graduandas }\end{array}$ & $\begin{array}{c}\text { Residência em } \\
\text { Ginecologia e obstetricia }\end{array}$ & $\%$ \\
\hline $1957-1960$ & 175 & 02 & 1,14 \\
\hline $1961-1970$ & 788 & 61 & 7,74 \\
\hline $1971-1980$ & 919 & 120 & 13,05 \\
\hline $1981-1990$ & 850 & 109 & 12,82 \\
\hline $1991-2001$ & 1060 & 117 & 11,03 \\
\hline
\end{tabular}


Atualmente, o Programa de Residência Para o Terceiro Ano (R3) é oferecido integralmente dentro das subespecialidades, designadas como Setores de Reprodução Humana; Gestação de Alto Risco e UltraSonografia; Oncologia Tocoginecológia e Mamária; e, Uroginecologia e Cirurgia Ginecológica.Junto ao Programa anual do R3, o departamento recebe um grande número de Médicos Estagiários que complementam sua formação dentro de uma das subespecialidades citadas anteriormente. Além disso, recebe Médicos Adidos, para reciclagem dentro de seus setores específicos, em programas com o mínimo de dois e o máximo de seis meses. Com essa oportunidade, os profissionais podem reciclar conhecimentos e inteirar-se dos avanços dentro da especialidade cumprindo um dos objetivos da Universidade, que é a difusão da prática do conhecimento, sem dúvidas, beneficiando a Sociedade.

Outra forma de difusão de conhecimento, largamente utilizada no Departamento de Ginecologia e Obstetrícia, é a que se baseia nos freqüentes Cursos de Atualização e Especialização, dirigidos mais aos profissionais da região, que se beneficiam dessa educação continuada e cujo resultado é altamente benéfico em todos os sentidos.

Até o ano de 2001, 154 residentes terminaram o programa de dois anos, dos quais 105 fizeram também o terceiro ano da especialidade (Tabela II).

\subsection{Ensino de Pós-Graduação Stricto Sensu}

Na evolução histórica do departamento, sobressai a década de 70 com a implantação do Curso de Pós-Graduação stricto sensu, na FMRP-USP e na área de Tocoginecologia. Foi um marco incontestável do desenvolvimento científico do nosso departamento,

\begin{tabular}{|cccc|}
\hline $\begin{array}{l}\text { Tabela II - Número de residentes de primeiro (R 1) } \\
\text { segundo (R2) e terceiro (R3) anos, do Departamento } \\
\text { de Ginecologia e Obstetricia de acordo com a } \\
\text { década de conclusăo do curso }\end{array}$ \\
\hline Ano & $R 1$ & $R 2$ & $R 3$ \\
\hline $1960-1970$ & 063 & 039 & 003 \\
\hline $1971-1980$ & 120 & 109 & 003 \\
\hline $1981-1990$ & 136 & 135 & 045 \\
\hline $1991-2001$ & 154 & 154 & 105 \\
\hline
\end{tabular}

visto que a ampliação dos seus campos de ensino pósgraduado e pesquisa sempre foram objetivos incansavelmente almejados. A criação do Curso de Pós-Graduação stricto sensu (Área de Tocoginecologia) não só veio de encontro a essa aspiração, como também abriu novos horizontes quanto à possibilidade de serem encontrados elementos capacitados para a docência e pesquisa. A possibilidade do pós-graduando cursar disciplinas dentro e fora da área, além de completar sua formação profissional, amplia sobremaneira seus conhecimentos em outros setores além daqueles da especialidade.

O Programa de Pós-Graduação em Tocoginecologia (Mestrado e Doutorado) foi autorizado pelo Magnífico Reitor da Universidade de São Paulo em 06/01/71, regido pela Portaria GR - 1212/70 de 25/06/70 da FMRP-USP. O curso foi reconhecido em 29/06/71 pelo Conselho Nacional de Pesquisa (CNPq), como Centro de Excelência, em condições de ministrar ensino pós-graduado em Tocoginecologia. O Mestrado e o Doutorado foram credenciados no Conselho Federal de Educação, em 1974, por um período de 5 anos, tendo, como área de concentração, a Tocoginecologia. Na oportunidade, o Chefe do Departamento de Ginecologia, Obstetrícia e Pediatria era o Prof. Dr. Alberto Raul Martinez e a Coordenação do Curso de Pós-Graduação da Tocoginecologia estava a cargo do Prof. Dr. Roberto Salles Meirelles. Entre 1978 e 1980, o Prof. Meirelles foi também o Vice-Presidente da Comissão de Pós-Gradução da FMRP-USP.

Os primeiros alunos matriculados no Programa de Pós-Graduação do Departamento foram os Drs. Sérgio Pereira da Cunha, Silésio do Prado, José Gonçalves Franco Jr. e Dr ${ }^{\mathrm{a}}$. Marta Edna H. Diógenes Yazlle. Posteriormente, todos eles foram contratados como professores do departamento.

Na década de 90, consolidou-se o Programa de Pós-Graduação da Área de Tocoginecologia do Departamento de Ginecologia e Obstetrícia, em decorrência da nítida especialização do seu corpo docente. Não se contratou mais nenhum docente sem conhecimento específico e sem o título de Doutor. Nessa década, formaram-se 37 Mestres e 19 Doutores em Tocoginecologia, conforme dados da Tabela III. Muitos dos alunos pós-graduados na Área de Tocoginecologia voltaram para suas instituições de origem após o término do curso, outros foram indicados para docentes em nosso departamento.

O Programa de Pós-Graduação do Departamento de Ginecologia e Obstetrícia da FMRP-USP 


\begin{tabular}{|c|c|c|}
\hline \multicolumn{3}{|c|}{$\begin{array}{l}\text { Tabela III - Número de Mestres e Doutores oriundos } \\
\text { do Prodrama de Pós-Graduacäo do Debartamento } \\
\text { de Ginecolodia e Obstetrícia da FMRP-USP seaundo } \\
\text { a décadla de conclusăo. }\end{array}$} \\
\hline \multicolumn{3}{|c|}{$N^{\circ}$ de profissionais titulados } \\
\hline Período & Mestrado & Doutorado \\
\hline $1958-1960$ & - & - \\
\hline $1961-1970$ & - & 3 \\
\hline $1971-1980$ & 24 & 16 \\
\hline $1981-1990$ & 37 & 19 \\
\hline $1991-2001$ & 74 & 45 \\
\hline Total & 135 & 83 \\
\hline
\end{tabular}

foi coordenado pelo Prof. Meirelles, de janeiro de 1971 até 1983; de 1983 até 1990, pelo Prof. Marcos Felipe; de 1990 a 1991, pelo Prof. Rui Ferriani; e, de março de 1991 até a presente data, pela Prof ${ }^{a}$. Maria Matheus.

Nos últimos cinco anos, a pós-graduação brasileira vem passando por uma notável reestruturação mediada pela Coordenação de Aperfeiçoamento de Pessoal de Nível Superior (CAPES), notadamente na Área Médica e na Área Biológica. Com a nova visão, tenta-se valorizar os programas com bom desempenho, reconhecendo-se, através de pontuações diferentes, a dimensão global de cada um deles. Embora não haja consenso sobre os instrumentos utilizados na avaliação, deve-se reconhecer que ela é necessária para evitar a banalização que a pós-graduação, vem sofrendo nessas áreas. Desde sua criação, as avaliações do Curso de Pós-Graduação do Departamento de Ginecologia e Obstetrícia são extremamente favoráveis, refletindo o bom desempenho tanto científico quanto o de formação pessoal do programa. Tais avaliações promovem uma reflexão contínua sobre a qualidade e desempenho do programa, fomentando mudanças e adaptações dirigidas para o aperfeiçoamento e o progresso acadêmico. Atualmente, o Programa de Pós-Graduação do citado departamento conta com 28 linhas de pesquisa e 28 disciplinas.

\subsection{Pós-Doutorado e Estágios de Curta Dura- ção no Exterior}

O Curso de Pós-Graduação no departamento, antes de e após sua instalação, trouxe, como conseqüência lógica, a necessidade de realização de Cur- sos de Pós-Doutorado e de Estágios de Curta Duração no Exterior, visando o aprimoramento e à diferenciação técnica/científica de seus docentes.

\subsection{Pós-Doutorado no Exterior}

O Prof. Roberto Salles Meirelles foi o primeiro a fazer Curso de Pós-Doutorado no Exterior, sendo agraciado com bolsa de estudo da Ford Foundation para tal finalidade. A partir de 15/04/68, ele fez o Curso Latino-Americano de Biologia de La Reproducción, realizado na Argentina, Uruguai e Chile. Esse curso possibilitou ao Prof. Meirelles aprofundar seus conhecimentos sobre esteroidogênese e estimular, com entusiasmo, o crescimento do Setor de Endocrinologia Ginecológica do Departamento.

Entre agosto de 1977 e agosto de 1978, a Prof ${ }^{a}$. Marta Edna Holanda Diógenes Yazlle, recebeu bolsa de Pós-Doutorado da Organização Panamericana de Saúde, para fazer seu estágio com os Profs. Ernesto Bojalil e Ramon Villareal, na Universidade Autônoma Metropolitana, Cidade do México, Distrito FederalMéxico. A aplicação dos conhecimentos adquiridos sobre ensino e assistência descentralizada, nortearam a futura atuação da Prof ${ }^{a}$ Marta nos Setores de Medicina Comunitária e de Anticoncepção.

O Programa de Pós-Doutorado no Exterior, desenvolvido pelo Prof. Marcos Felipe de Sá, foi o primeiro executado dentro dos modernos objetivos desse tipo de programa, ou seja, ligado a um projeto de pesquisa específico, com início, meio e fim. Ele recebeu bolsa da FAPESP para trabalhar no Serviço do Prof. Samuel Yen, na University of California, San Diego, California-USA, no período de 01/02/79 a 20/ 12/80. A experiência e o conhecimento adquiridos no Curso de Pós-Doutorado, facilitaram o trabalho do Prof. Felipe após seu retorno, fazendo crescer objetivamente o Setor de Endocrinologia Ginecológica e o Laboratório do Departamento de Ginecologia e Obstetrícia. Na sequiência, todos os docentes do departamento que fizeram pós-doutorado no Exterior desenvolveram projetos inteiros dentro de seus programas.

O Prof. Rui Alberto Ferriani fez Pós-Doutorado na University of Cambridge, Cambridge-Inglaterra, de 06/04/91 a dezembro de 1992, recebendo bolsa do CNPq. Seu projeto sobre mediação química na esteroidogênese foi desenvolvido no Serviço do Prof. Stephen Smith, revertendo em conhecimento diferenciado e numerosas publicações em periódicos de alto impacto editorial. Com a volta do Prof. Rui, o Setor de Reprodução Humana foi objetivamente enriquecido. 
Em julho de 1993, o Prof. Marcos Dias de Moura iniciou seu Programa de Pós-Doutorado no Exterior, o qual foi desenvolvido na University of Maryland, Maryland, Baltimore-USA,até maio de 1996. Inicialmente, sua bolsa foi financiada pelo CNPq e, depois, pela FAPESP. Seu projeto de pesquisa sobre esteroidogênese ovariana foi desenvolvido no Serviço do Prof. Eli Adashi e foi considerado precursor para várias outras linhas de pesquisa. Na sua volta, o Prof. Marcos retornou com suas atividades junto ao Setor de Reprodução Humana, seu grupo de origem.

O Programa de Pós-Doutorado no Exterior, realizado pelo Prof. Geraldo Duarte, foi desenvolvido na University of Pittsburgh, Pittsburgh, PennsylvaniaUSA, entre 31/07/1997 a 31/07/1999, com bolsa financiada pela FAPESP. No período mencionado, ele trabalhou no Serviço do Prof. Daniel Vincent Landers, desenvolvendo projeto sobre transmissão vertical do HIV e Gestação de Alto Risco. Após seu retorno, vem tentando aplicar o conhecimento que adquiriu na condução de pesquisas ligadas ao cuidado de gestantes infectadas pelo HIV e gestantes portadoras de algum risco materno/perinatal.

\subsection{Estágios de Curta Duração no Exterior}

- Prof ${ }^{a}$. Maria Matheus de Sala estagiou no Serviço do Prof.Hermógenes Alvares. Laboratório de Investigaciones en Reproducción Humana do Hospital Pereira Rossel, Montevideu-Uruguai, em 1973;

- Prof. Sérgio Bighetti estagiou no Serviço do Prof. Roberto Esteves. Hospital Militar, Buenos AiresArgentina, em 1975;

- Prof ${ }^{a}$. Maria Matheus de Sala estagiou com os Profs. John Rock e Rene Genadry durante o Primer Curso Latinoamericano de Microcirurgia Tubárica, organizado pela Johns Hopkins Medical Institution e Ministério de Salud de Colombia. BogotáColombia, em 1980;

- Prof. Sérgio Bighetti estagiou na Universidade de Stanford. Stanford-USA, em 1980;

- Prof. Sérgio Pereira da Cunha estagiou na Universidade Johns Hopkins. Baltimore-USA, em 1982;

- Prof. Sérgio Pereira da Cunha estagiou no Centers for Disease Control (CDC) dos Estados Unidos da América. Atlanta-USA, em 1982;

- Prof. Sérgio Bighetti estagiou no Hospital MD Anderson Cancer Center. Universidade do Texas, Houston-USA, em 1985;
- Prof ${ }^{a}$. Maria Matheus de Sala estagiou no Serviço dos Profs. Pedro Barri e Santiago Dexeus. Laboratório de Investigaciones en Reproducción $\mathrm{Hu}$ mana do Instituto Dexeus. Universidad Autonoma de Barcelona, Barcelona-Espanha, em 1986;

- Prof. Francisco Mauad Filho estagiou no Serviço do Prof. Fernando Bonilla-Mussoles. Hospital Universitario de Valencia-Espanha, em 1987;

- Prof. Marcos Felipe Silva de Sá estagiou no Serviço do Prof. Fernando Bonilla-Mussoles. Hospital Universitário de Valencia, Valencia-Espanha, em 1988;

- Prof. Geraldo Duarte estagiou no Serviço do Prof. Fernando Bonilla-Mussoles. Hospital Universitario de Valencia, Valencia-Espanha, em 1991;

- Prof. Marcos Dias de Moura estagiou no Serviço do Prof. Fernando Bonilla-Mussoles. Instituto Valenciano de Infertilidad, Valencia-Espanha, em 1992;

- Prof. Rui Ferriani estagiou no Serviço do Prof. Eli Adashi. Universidade de Maryland, Baltimore-USA, em 1994;

- Prof. Geraldo Duarte estagiou no Serviço dos Prof. Stephen Morse. Centers for Disease Control and Prevention (CDC) dos Estados Unidos da América, Atlanta-USA, em 1994; e

- Prof. Aderson Tadeu Berezowski estagiou no Serviço do Prof. Kypros Nicolaides. Kings College School of Medicine, Londres-Inglaterra, em 1997.

\subsection{Assistência}

A assistência à comunidade prestada pelo Departamento de Ginecologia e Obstetrícia da FMRPUSP já foi efetuada em várias instituições, ou seja, Hospital das Clínicas (antiga Maternidade Sinhá Junqueira), na Santa Casa de Misericórdia de Ribeirão Preto, no Hospital das Clínicas-Campus, na Unidade de Emergência, na Mater, no Posto de Saúde de Vila Lobato e no Centro de Saúde-Escola.

O primeiro parto no Departamento de Ginecologia e Obstetrícia foi normal e realizado pelo Dr. Roberto Luis Nogueira, às 13:40h do dia 07/08/56. A parturiente foi a Sra. Durvalina Lúcio Tavares*, que deu a luz a um recém-nascido do sexo masculino, em boas condições de saúde. A primeira cirurgia no departamento, foi realizada pelo Dr. José de Carvalho Guimarães, às 10:00 horas do dia 31/08/56. A paciente IR foi submetida a uma colpoperineorrafia anterior e posterior, com raquianestesia, na sala 03 , do Centro

\footnotetext{
* Autorização concedida para publicação do nome, ao Chefe do Departamento
} 
Cirúrgico do Hospital das Clínicas da Bernardino de Campos (atual Unidade de Emergência).

Com base nos relatórios de atendimento, conclui-se que a assistência médica no Departamento de Ginecologia e Obstetrícia veio crescendo desde o início de sua implantação até os dias atuais. A Região de Ribeirão Preto se desenvolveu rapidamente e o crescimento populacional foi um dos fatores importantes para o aumento da demanda na prestação de serviços. De tempos em tempos, o departamento teve períodos de estrangulamento, com excesso de pacientes e pouco espaço, com sobrecarga para o pessoal médico e paramédico responsáveis pelo ensino, assistência e pesquisa.

No início, o setor contou com a colaboração dos Médicos Assistentes Voluntários e, posteriormente, com os Médicos Assistentes do Hospital das Clínicas, prestando relevantes serviços à comunidade. Cronologicamente, as Chefias de Clínica do Departamento estiveram a cargo dos seguintes professores: Domingos Andreucci (1956-1958); Bussamara Neme (maio a novembro de 1958); Alberto Martinez (1958-1960); Ítalo Baruffi (1961-1978); Sérgio Pereira da Cunha (1978-1988); Geraldo Duarte (1988-1997); e Antonio Alberto Nogueira (1997-até o momento).

Sempre trabalhando com o objetivo de cumprir ensino, assistência e pesquisa, o departamento viu que, sem uma assistência bem estruturada, não haveria como cumprir adequadamente os outros dois objetivos. Então, ao lado do atendimento básico, foi necessário ampliar as fronteiras das subespecialidades, atendendo não apenas a pesquisa, mas também a demanda de mercado profissional por tocoginecologistas preparados de acordo com o progresso técnico e científi- co da atualidade. Atendendo a esses quesitos, o departamento vem ampliando seguidamente seus setores especializados, que, atualmente, já são 15.

A sinopse numérica da assistência prestada pelo Departamento de Ginecologia e Obstetrícia pode ser observada na Tabela IV.

\subsection{Pesquisa}

A partir de 1960, a pesquisa científica, que era frágil, começou a estabelecer bases sólidas através de intercâmbios mantidos com outras áreas da FMRPUSP, principalmente as básicas. Inicialmente, estabeleceu-se a relação com os vários departamentos da unidade e, posteriormente, com outras instituições nacionais e internacionais (Escola de Fisiologia Obstétrica do Prof. Caldeyro Barcia-Uruguai; Instituto de Biologia y Medicina Experimental Dr. Bernard Houssay-Argentina; Prof. Bonilla-Espanha; Prof. YenEUA; Prof. Smith-Inglaterra; Prof. De Ziegler-França; Prof. Adashi-USA; Prof. Daniel Landers-USA, Profa. Sharon Hillier-USA, entre outros). Esses objetivos foram delineados sob a chefia do Prof. Martinez, e mantidos até a presente data com o Prof. Geraldo, passando pelos Professores Ítalo Baruffi, Roberto Salles Meirelles, Marcos Felipe de Sá, Sérgio Pereira da Cunha e Rui Ferriani. As relações mantidas com outros departamentos da FMRP e com instituições estrangeiras, vieram sedimentar os conhecimentos na área e permitir a formação de docentes de alto nível profissional, bem como docentes pesquisadores altamente qualificados para a pesquisa científica, que se estabeleceram não só na Universidade de São Paulo, como, também, nas demais faculdades em diferentes regiões do País.

Tabela N - Sinopse numérica das atividades assistenciais prestadas no Departamento de Ginecologia e Obstetricia, segundo a década de realizacão dos procedimentos

\begin{tabular}{|lccccc|}
\hline \multicolumn{1}{|c}{ Procedimentos } & \multicolumn{5}{c|}{ Períodos } \\
\hline & $1958-1960$ & $1961-1970$ & $1971-1980$ & $1981-1990$ & $1991-2001$ \\
\hline Internaçốes & 2.772 & 42.670 & 36.050 & 41.110 & 58.717 \\
\hline Consultas & 17.559 & 132.300 & 322.310 & 421.049 & 469.249 \\
\hline Partos & 1.719 & 25.660 & 19.770 & 32.200 & 36.593 \\
\hline Cirurgias & 1.704 & 2.645 & 4.590 & 5.001 & 12.226 \\
\hline Ex. Subsidiários & - & - & $16.535^{*}$ & 160.833 & 168.637 \\
\hline *Transterência para o Hospital das Clínicas-Campuse início das atividades do Laboratório de Tocoginecologia
\end{tabular}


A Tabela $\mathrm{V}$ relaciona a produção científica da área, representando, assim, os resultados obtidos após 45 anos de existência.

Além das atividades citadas, o Departamento de Ginecologia e Obstetrícia tem participado intensamente em congressos, jornadas, símpósios e reuniões científicas, no Brasil e no Exterior. Ele teve produção científica substancial, representada por mais de 800 trabalhos publicados em revistas nacionais e estrangeiras, muitos deles agraciados com premiações nacionais e internacionais, colocando em evidência o nome do departamento.

\section{CONTRIBUIÇÃO DO DEPARTAMENTO DE GINECOLOGIA E OBSTETRICIA EM ATIVIDADES ACADÊMICAS E ADMINIS- TRATIVAS EXTRADEPARTAMENTAIS}

Paralelamente às atividades intradepartamentais, os docentes do Departamento de Ginecologia e Obstetrícia têm participado ativamente da vida acadêmica, universitária e das sociedades médicas, colaborando para o funcionamento e aperfeiçoamento de nossas instituições. Nesse contexto, relembre-se que o Prof. Martinez foi Superintendente do Hospital das Clínicas, Diretor da FMRP-USP e da Escola de Enfermagem de Ribeirão Preto-USP. Destaca-se, também, a contribuição de outros dois docentes do departamento na Superintendência do Hospital das Clínicas, o Prof. Martinelli e o Prof. Marcos Felipe. O Prof. Martinelli dirigiu essa instituição por 12 anos consecutivos e o Prof. Marcos Felipe é o Superintendente desde 1995. Destaca-se, também, a Vice-Coordenadoria da Comissão de Pós-Graduação da FMRP-USP exercida pelo Prof. Meirelles.

\begin{tabular}{|ccc|}
\hline $\begin{array}{l}\text { Tabela V } \\
\text { docentes do Número de trabalhos publicados por } \\
\text { Obstetricia de acordo como periodo em anos }\end{array}$ \\
\hline \multicolumn{3}{|c|}{ Trabalhos Publicados } \\
\hline $\begin{array}{c}\text { Periodo em } \\
\text { Anos }\end{array}$ & $\begin{array}{c}\text { Revistas } \\
\text { nacionais }\end{array}$ & $\begin{array}{c}\text { Revistas } \\
\text { internacionais }\end{array}$ \\
\hline $1958-1960$ & 03 & - \\
\hline $1961-1970$ & 39 & 03 \\
\hline $1971-1980$ & 112 & 30 \\
\hline $1981-1990$ & 272 & 55 \\
\hline $1991-2001$ & 251 & 83 \\
\hline TOTAL & 677 & 171 \\
\hline
\end{tabular}

Os editores de três importantes periódicos científicos são docentes do Departamento de Ginecologia e Obstetrícia. O Prof. Greene é o Editor do Brazilian Journal of Medical and Biological Research; o Prof. Jurandyr é o Editor da Revista Brasileira de Ginecologia e Obstetrícia; e o Prof.Rui é o Editor da Revista Reprodução e Climatério. O Prof.Geraldo é Editor Associado do Jornal Brasileiro de Doenças Sexualmente Transmissíveis. São contribuições que, sem dúvidas, distinguem e elevam o nome do departamento.

A criação da FAEPA, em 1988, possibilitou criar mecanismos mais ágeis de apoio aos departamentos e aos docente em RDIDP, alternativas essenciais ao financiamento de pequenos projetos e à manutenção e ampliação departamental na FMRP-USP. Desde o início, a participação dos nossos docentes, na citada fundação, tem sido de extrema importância, visto que seu primeiro Diretor Executivo foi o Prof. Marcos Felipe e, atualmente, o Prof. Rui que é seu Diretor Científico.

O destaque do Departamento de Ginecologia e Obstetrícia, nas Sociedades Médicas de Ginecologia e Obstetrícia, tem sido relevante ao longo do tempo. No campo da Oncologia, destaca-se o papel do Prof. Baruffi, um dos idealizadores e criadores da Sociedade Brasileira de Colposcopia e Patologia do Trato Genital Inferior. Da mesma forma, a criação da Sociedade Interiorana de Ginecologia e Obstetrícia do Estado de São Paulo, cuja liderança foi dos docentes do departamento. Os Profs. Marcos Felipe e Rui Ferriani foram os criadores da Sociedade Brasileira de Ginecologia Endócrina (SOBRAGE).

O Prof. Sérgio Cunha exerceu a Presidência e Vice-Presidência (duas vezes) da Sociedade de Obstetrícia e Ginecologia do Estado de São Paulo 'residência da Federação Braside Ginecologia e Obstetrícia zríodo de intensa atividade proa. O primeiro Presidente da If. Marcos Felipe e, na seqüên-

ões Nacionais da FEBRASGO ordenação direta ou indireta de rento de Ginecologia e ObstetríCoordenações de Uroginecolo:ício; a de Ginecologia Endócrise; a de Reprodução Assistida, : Gestação de Alto Risco e a de a, com o Prof. Sérgio. Por dois 
mandatos consecutivos, o Prof. Geraldo foi Vice-Presidente da Comissão Nacional de Doenças Sexualmente Transmissíveis/AIDS, sendo o seu Presidente na atualidade. Participar ativamente dessas comissões transcende $\mathrm{o}$ aspecto político, pois significa a chance objetiva para desencadear mudanças e transformações em prol do crescimento profissional e acadêmico no país.

Esta é a segunda publicação que aborda a evolução histórica do Departamento de Ginecologia e Obstetrícia da FMRP-USP. A seguir, dados da bibliografia referente ao primeiro documento publicado: Meirelles RS. Dados históricos, relativos à implanta- ção, ao desenvolvimento e às atividades do Departamento de Ginecologia e Obstetrícia da Faculdade de Medicina de Ribeirão Preto da Universidade de São Paulo. Medicina, Ribeirão Preto, 25: 94-103, 1992.

\section{AGRADECIMENTOS}

Neste documento, que resgata a história do Departamento de Ginecologia e Obstetrícia, agradecemos aos Profs. Alberto Raul Martinez, Carlos Eduardo Martinelli, Maria Matheus de Sala, Lewis Joel Greene e Sergio Pereira da $\mathrm{Cu}-$ nha. Nossos agradecimentos também à Senhorita Iara Maria Corrêa por sua contribuição na recuperação de dados. 